\title{
$O$ controle social e o direito à saúde das mulheres
}

\author{
Social control and women's right to health \\ Control social y derecho de la mujer a la salud
}

Recebido: 15/10/2021 | Revisado: 22/10/2021 | Aceito: 25/10/2021 | Publicado: 29/10/2021

\author{
Marcella Neiva Romero \\ ORCID: https://orcid.org/0000-0001-9581-3984 \\ Faculdade de Ciências Médicas da Santa Casa de São Paulo, Brasil \\ E-mail: mnromero.mnr@gmail.com \\ Gabriela Almeida Damasio \\ ORCID: https://orcid.org/0000-0002-5599-6728 \\ Faculdade de Ciências Médicas da Santa Casa de São Paulo, Brasil \\ E-mail: gabikauzilaalmeida@gmail.com \\ Maria Fernanda Terra \\ ORCID: https://orcid.org/0000-0003-1718-4216 \\ Faculdade de Ciências Médicas da Santa Casa de São Paulo, Brasil \\ E-mail: mfterra@gmail.com
}

\begin{abstract}
Resumo
Este artigo buscou analisar como as militantes da União de Mulheres de São Paulo compreendem a garantia do direito à saúde das mulheres a partir da Atenção Primária à Saúde. Para esse estudo, foi utilizado o método qualitativo, a partir de entrevistas semiestruturadas com três militantes da Organização Não Governamental União de Mulheres, no município de São Paulo. As entrevistas foram analisadas a partir da técnica de análise de conteúdo de Bardin. Do material empírico produzido, foram trabalhados como parte da categoria de análise: barreira no acesso à saúde. As mulheres conhecem e militam acerca do acesso à direitos, pois duas delas participaram da Construção do Programa de Atenção Integral à Saúde das Mulheres (PAISM). Elas apontam fragilidades no sistema a ser trabalhado sob a perspectiva da humanização como caminho para a garantia dos direitos das mulheres. Faz-se necessário revisitar as práticas cotidianas dos profissionais de saúde na assistência às mulheres, de maneira a identificar e combater preconceitos e julgamentos morais que negam direitos, além de impactarem negativamente a vida das mulheres.
\end{abstract}

Palavras-chave: Feminismo; Saúde da mulher; Direito da mulher.

\begin{abstract}
This article sought to analyze how the activists of the Union of Women of São Paulo understand the guarantee of the right to women's health from Primary Health Care. For this study, the qualitative method was used, based on semistructured interviews with three militants of the Non-Governmental Organization Union of Women, in the city of São Paulo. The interviews were analyzed using Bardin's content analysis technique. Of the empirical material produced, they were worked as part of the category of analysis: barrier in access to health. Women know and militate about access to rights, because two of them participated in the Construction of the Comprehensive Health Care Program for Women (PAISM). They point to weaknesses in the system to be worked from the perspective of humanization to guarantee women's rights. It is necessary to revisit the daily practices of health professionals in the care of women, to identify and combat prejudices and moral judgments that deny rights, besides negatively impacting women's lives.
\end{abstract}

Keywords: Feminism; Women's health; Women's law.

\section{Resumen}

Este artículo buscó analizar cómo las activistas de la Unión de Mujeres de São Paulo entienden la garantía del derecho a la salud de las mujeres desde la Atención Primaria de Salud. Para este estudio se utilizó el método cualitativo, basado en entrevistas semiestructuradas a tres militantes de la Organización No Gubernamental Unión de Mujeres, en la ciudad de São Paulo. Las entrevistas fueron analizadas utilizando la técnica de análisis de contenido de Bardin. Del material empírico producido, se trabajaron como parte de la categoría de análisis: barrera en el acceso a la salud. Las mujeres conocen y militan sobre el acceso a los derechos, porque dos de ellas participaron en la Construcción del Programa de Atención Integral a la Salud de las Mujeres (PAISM). Señalan debilidades en el sistema a trabajar desde la perspectiva de la humanización como una forma de garantizar los derechos de las mujeres. Es necesario revisar las prácticas cotidianas de los profesionales de la salud en el cuidado de las mujeres, con el fin de identificar y combatir los prejuicios y juicios morales que niegan derechos, además de impactar negativamente en la vida de las mujeres.

Palabras clave: Feminismo; Salud de la mujer; Derecho de la mujer. 


\section{Introdução}

O movimento feminista luta pelos direitos das mulheres reivindicando igualdade no acesso a direitos em todos os momentos da vida, desde a participação na política, vida social e econômica, como no acesso ao trabalho bem remunerado, saúde, moradia, autonomia e liberdade de expressão (Paradis, 2014). O movimento feminista contribuiu para a criação e implantação do Programa de Atenção Integral a Saúde das Mulheres (PAISM ${ }^{1}$ ), em 1983, e, posteriormente, na construção dos pilares do Sistema Único de Saúde (SUS), em 1990 por meio da promulgação da Lei n 8.080/90 (Brasil, 1990; Souto \& Moreira, 2021). A importância do movimento social está descrita na Lei Orgânica da Saúde que subsidia a implementação do SUS, através do princípio da participação social e do controle social do SUS (Brasil, 2015).

A partir da compreensão de que a participação social é fundamental para assegurar direitos e, posteriormente, efetiválos. Santos (1987) endossa essa questão, ao referir que as propostas de políticas públicas não se consolidam sem a participação popular na sua construção, supervisão e cobrança para a sua efetivação, principalmente no contexto econômico, que se baseia no ganho de capital e reprodução das desigualdades a partir do discurso da meritocracia. Nesse contexto, devido às desigualdades impostas às mulheres, principalmente às mulheres negras e pobres, faz-se necessário refletir que práticas e políticas públicas de saúde se efetivam para a população de mulheres atendidas nessas instituições, principalmente, pelo fato da maioria delas serem pobres, negras e não participantes do sistema de produção capitalista reproduzido na sociedade e, por isso, faz-se necessário cobrar e acompanhar o desenvolvimento de políticas que reconheçam tais contextos de vulnerabilidade. A partir desse referencial, este estudo busca identificar como as feministas da ONG União de Mulheres reconhecem a garantia dos direitos à saúde das mulheres a partir da assistência nos serviços de Atenção Primária à Saúde (APS) (Santos, 1987).

\section{Metodologia}

Foi realizada uma pesquisa qualitativa, tendo por material empírico as entrevistas com feministas, participantes da Organização Não Governamental (ONG) União de Mulheres de São Paulo. A análise do objeto de estudo, as percepções de militantes acerca da garantia dos direitos em saúde das mulheres se pautam no referencial teórico da participação social como fundamental para a efetivação dos direitos. A pesquisa foi submetida e aprovada pelo conselho consultivo da União de Mulheres de São Paulo e, posteriormente, pelo Comitê de Ética em Pesquisa (CEP) da Santa Casa de São Paulo. Os dados empíricos foram coletados por meio de três entrevistas semiestruturadas, gravadas e com posterior transcrição integral, realizadas após a leitura e assinatura do Termo de Consentimento Livre e Esclarecido (TCLE). A pesquisa foi aprovada com sob o código na Plataforma Brasil: CAAE: 56141416.6.0000.5479. Utilizou-se da técnica de Análise de Conteúdo proposta por Bardin (1977), para decodificar o material empírico, e estabelecer as categorias de análise.

A técnica de análise de conteúdo é uma metodologia analítica que se organiza em fases para que os dados coletados sejam analisados:1. Pré-análise: "leitura flutuante" para compreender como os conteúdos aparecem para responder ao objetivo do estudo. 2. Exploração do material: nessa etapa, serão definidos os temas, ou seja, quais foram as informações pertinentes que apareceram e que respondem ao objetivo do estudo. 3. Tratamento dos resultados obtidos e interpretação: envolve a interpretação dos resultados encontrados e, a partir do referencial teórico utilizado na pesquisa, serão criadas as dimensões teóricas de discussão dos resultados empíricos (Bardin, 1977).

\footnotetext{
${ }^{1}$ PAISM - Programa de Assistência Integral à Saúde das Mulheres.
} 


\section{Resultados e Discussão}

Foram entrevistadas três mulheres feministas que atuavam na Organização Não Governamental (ONG) União de Mulheres do Município de São Paulo. Seus nomes foram modificados para garantir o sigilo, e estão apresentadas no quadro abaixo.

Quadro 1: Apresentação das mulheres entrevistadas da Organização Não Governamental - União de Mulheres, 2015.

\begin{tabular}{|lcll|}
\hline $\begin{array}{c}\text { Identificaçã } \\
\text { o }\end{array}$ & Idade & formação & \multicolumn{1}{c|}{ Relação com o movimento de mulheres e União de Mulheres } \\
\hline Anne Fisher & 77 & $\begin{array}{l}\text { Bacharel em } \\
\text { direito }\end{array}$ & $\begin{array}{l}\text { Pioneira na União de Mulheres. Foi presa política, torturada na frente de seus } \\
\text { filhos durante a ditadura militar. Participou da construção do PAISM } \\
\text { de } 80 \text { e atualmente, participa na forcada } \\
\text { humanos. }\end{array}$ \\
\hline $\begin{array}{l}\text { Kathrine } \\
\text { Switzer }\end{array}$ & 75 & Enfermeira & $\begin{array}{l}\text { Atuou como enfermeira na guerrilha do Araguaia; foi presa política e torturada } \\
\text { durante a ditadura militar grávida, deu à luz na prisão e foi impedida de ver o } \\
\text { filho por } 53 \text { dias. Participou da construção do PAISM e participa na formação } \\
\text { política de mulheres em direitos humanos. }\end{array}$ \\
\hline $\begin{array}{l}\text { Leola } \\
\text { King }\end{array}$ & $\mathrm{N}$ & Advogada & $\begin{array}{l}\text { Militante LGBTQIA+ e integrante do movimento União de Mulheres desde } \\
\text { 2010. Participou na formação política de mulheres em direitos humanos. }\end{array}$ \\
\hline
\end{tabular}

Fonte: Pesquisadora (2015).

A partir da análise do material empírico emergiram vários temas, como: aborto legal, direito sexual, direito reprodutivo, violência obstétrica, feminicídio, violência de gênero, falta de humanização e falta de formação em gênero pelos profissionais de saúde, que foram estruturados na seguinte categoria de análise:

\section{Barreira no acesso à saúde}

Foi abordada pelas entrevistadas a temática do aborto, como um direito não garantido, apesar da existência de uma lei no Brasil. Para a entrevistada Kathrine, o aborto é um tema fundamental para se pensar a garantia dos direitos das mulheres. Observou-se que, embora exista a lei 2.848 de 07/12/1940, que dispõe sobre a garantia do direito ao aborto legal no Brasil em situações específicas, ainda assim, esse o direito é pouco orientado e oferecido às mulheres (Brasil, 1940).

“\{...\} no serviço de saúde, nem mesmo o aborto previsto por lei, que é uma lei da década de 40... Nem este é feito no serviço de saúde. Hoje, se houver estupro nas grandes cidades, pode ser que a mulher ainda consiga um atendimento, mas se for risco de vida da mãe, não é, e a lei prevê em casos de risco de vida da mãe ou estupro \{...\} não é fácil. Mas risco de vida da mãe não, então as mulheres morrem de eclampsia, que certamente teriam indicação de fazer um aborto. Então essa é a questão do aborto, o movimento detectou que tinha até o direito legal que não era cumprido e batalhou por ele, mas não conseguiu plenamente, porque risco de vida da mãe vai depender da opinião de terceiros, do médico, e eu nunca vi ninguém recomendar aborto por risco de vida da mãe \{...\}”. - Kathrine Switzer

Essa percepção é corroborada pela literatura que reforça o contexto de moralidade e julgamento que permeia a prática assistencial às mulheres em situação de abortamento que chegam nos serviços de saúde, deixando o cuidado à vida das mulheres em segundo plano (Talib \& Citeli, 2005). O julgamento sofrido pelas mulheres que chegam em abortamento deveria ser reconhecido como condição de risco às mulheres, como descrito na Política Nacional de Atenção Integral à Saúde da 
Mulher (Brasil, 2004), que o aborto inseguro, caso tenha sido o realizado, pode causar morte às mulheres e que o não atendimento se configura como violência (Medeiros, 2021).

Apesar dos direitos, a existência de apenas 68 serviços cadastrados para essa prática, sendo que apenas 37 o realizam e, mesmo assim, o fazem mediante exigências não previstas na Lei, tais como: a apresentação de boletim de ocorrência, apresentação de um alvará judicial ou pericial aprovando o procedimento e/ou avaliação psiquiátrica da paciente (Diniz et al., 2014).

Segundo Talib e Citeli (2005) o acesso aos serviços de saúde é ainda pior às mulheres negras e moradoras das periferias (Cardoso et al., 2020). Para elas são ofertadas poucas tecnologias em saúde, como anestesia, medicamentos e orientações (Diniz et al., 2014). Assis (2018) aponta que a perpetração da violência obstétrica no Brasil demarca espaços sociais, não apenas pela condição socioeconômica, mas pela caracterização fenotípica do sujeito, que é usada para a reprodução de discriminação, preconceitos e exclusão (Sena \& Tesser, 2017).

Apesar do julgamento mais intenso sobre as mulheres jovens e negras, a literatura (Diniz et al., 2009) afirma que a Organização Pan-Americana da Saúde (OPAS) mostrou que o perfil de mulheres que fizeram aborto nos últimos 20 anos é composto por jovens com idades entre 20 e 29 anos, em união estável, com até oito anos de estudo, trabalhadoras, católicas, com pelo menos um filho e usuárias de métodos contraceptivos, as quais abortam com misoprostol (Madeiro \& Diniz, 2016).

Somado ao tema do aborto, aparecem nas narrativas o tema dos direitos sexuais e reprodutivos como pouco discutidos nas práticas em saúde sob a perspectiva da autonomia e liberdade de escolha das mulheres.

“\{...\} Foi um processo concomitante, por que eu venho da geração que pode usar a pílula anticoncepcional, foi a primeira geração, praticamente, dos anos 60. Uma geração em que o exercício da sexualidade vai ter mais liberdade do que as anteriores e eu acho que nós tivemos muita discussão a respeito da sexualidade, do exercício da sexualidade. $O$ direito ao orgasmo, que nós fomos discutir em público nos anos 70, porque nós descobrimos que muitas mulheres não tinham orgasmo, não tinham prazer sexual, tinham até nojo de fazer sexo, sentiam muita dor. Eram muitas tentativas e pouco prazer $\{\ldots\}$... - Anne Fisher

"Mas eu tô pensando na saúde das mulheres lésbicas, o quanto quando a gente vai às consultas em saúde e a abordagem é heteronormativa, que vai pressupor que essa mulher se relacione com um homem que tem pênis, limita eu de falar a respeito da minha sexualidade, e consequentemente de eu buscar esse cuidado com a minha saúde, por que, por exemplo, pensando no Papanicolau; explica-se qual é o intuito desse exame? Por que é importante fazer? E por que é importante fazer independente de eu me relacionar com um homem com uma mulher ou com ambos? 'É meio que abre a perna, eu vou enfiar esse negócio que vai machucar, por que eu não vou ter cuidado algum pra fazer isso'. Então enfia um troço que é muito mais invasivo e agressivo do que preventivo." - Leola N King

"Nós discutimos muito a maternidade. A maternidade é uma sobrecarga enorme, porque somos as únicas responsáveis, pela educação das crianças, embora de uma forma imposta. Na realidade toda a sociedade tinha que ter essa obrigação para com as crianças, mas recaem sobre nós mulheres; os homens ninguém cobra deles a ausência da paternidade. Nós discutimos então, a gravidez como obrigação, por que mulher tem que ficar grávida, assim como nós discutimos também o direito de não ficar grávida, de nunca ter filhos e sermos mulheres cidadãs, respeitadas e dignas como qualquer mulher, por que eu venho de um tempo de discussão, como é até hoje, a maternidade obrigatória $\{\ldots\}$, - Anne Fisher

“(...) eu sempre tive parto normal, parto sem dor, eu fiz relaxamento, eu fiz respiração, eu fiz exercício de expulsão por minha conta, orientada por um médico, mas por minha conta. Agora as mulheres têm que fazer cesariana, eles nem discutem... acho muito mais difícil ter um parto normal hoje do que no meu tempo. É excepcional. É violência." Anne Fisher 
É importante considerar que a sexualidade é uma dimensão da vida subjetiva, afetiva e relacional das pessoas e, portanto, precisa ser abordada com cuidado e a construção com as mulheres é de extrema importância. Os direitos sexuais e reprodutivos estão descritos na constituição e devem ser garantidos. O país assume essa responsabilização ao tornar-se signatário da Conferência Internacional Sobre População e Desenvolvimento, realizada no Cairo, em 1994, e da IV Conferência Mundial sobre a Mulher - Igualdade, Desenvolvimento e Paz, realizada em Pequim, em 1995 (Busin, 2013). Os direitos sexuais e reprodutivos são interdependentes, não similares, como abordam os serviços de saúde. Sobre isso, a entrevistada Anne relata que a década de 60 foi um período de luta pelo direito sexual pelas mulheres, principalmente com o lançamento da pílula anticoncepcional, marco na garantia da liberdade sexual das mulheres (Costa, 2009).

Nesse contexto, é dever da equipe realizar atividades que abordem essa temática nas Unidades Básicas de Saúde (UBS) de modo a contemplar mulheres de todas as idades, considerando os aspectos individuais de cada uma delas sem julgamentos ou preconceitos, permitindo a elas escolherem os métodos contraceptivos que desejam utilizar, ou não, como também garantir que tenham acesso a métodos de barreira pra a proteção de doenças, além de orientações sobre violência sexual e estratégias de enfrentamento, de modo a contribuir para que as experiências sexuais sejam consideradas e vividas plenamente, de acordo com o desejo de cada uma delas (Brasil, 2012).

Referindo-se a questão da orientação sexual (Brasil, 2014), que é tema pouco abordado nas práticas em saúde, as consultas se baseiam em uma assistência que massifica as mulheres sob uma abordagem heteronormativa (Santos et al., 2019). Dentre as falhas estão a não oferta de orientação a respeito do exame de Papanicolau para as mulheres lésbicas ou, quando não é negada a realização pelo fato de não se relacionarem sexualmente com os homens (Santos et al., 2010). Sobre os direitos reprodutivos, as entrevistadas relatam a sobrecarga da mulher quanto à maternidade, sob a perspectiva da responsabilidade e da sobrecarga imposta a elas.

A entrevistada traz a importância da maternidade para as mulheres; discussão corroborada pela literatura que, na perspectiva de gênero, reforça a postura multivalente da mulher, que é vítima do conceito de divisão sexual do trabalho imposto pela sociedade que, segundo Saffioti (1997), mesmo em função do capital "não equaliza todas as forças de trabalho.". As mulheres são consideradas historicamente como as responsáveis pela criação e educação dos filhos, somado ao cuidado das demandas da casa, além dos seus trabalhos formais, enquanto os homens, colocados como os líderes na hierarquia social, desfrutam da liberdade conquistada pela sociedade patriarcal (Saffioti, 1997; 2011).

Na prática assistencial, apesar da discussão de gênero em protocolos assistenciais, as orientações focam na assistência ao biológico durante o pré-natal, parto e puerpério, desconsiderando os determinantes sociais, assim como as questões de classe social e gênero (Viviane et al., 2020).

Segundo Diniz, Cabral e Santos (2004), as mulheres são pouco ouvidas e o tema da vivência do corpo e da sexualidade pouco abordado (Brasil, 2016). Esses temas são parte dos direitos reprodutivos, que envolvem o dever em assegurar o direito da mulher de decidir, se pretende ter filhos e o momento ideal de concebê-los, permitindo que ela possa escolher um método contraceptivo, ou não, sem distingui-la por idade, estado civil e encorajando-a sobre seu poder de escolha sobre si mesma e seu corpo (Silva, 2013).

Sobre o recorte de gênero, Anne Fisher fala sobre a impossibilidade de dialogar sobre o parto e sobre a posição impotente na qual a mulher é posta nesse momento, que deveria ser de autonomia; isso devido a relação de desigualdade a qual a mulher foi posta na sociedade que foi construída baseada no patriarcado (Tesser et al., 2015). Desse modo, a mulher é vista como um ser vulnerável, que circula na sociedade possuindo menos poder que os homens, o que legitima ações de abuso contra elas (Weidle et al., 2014; Bourguignon \& Grisotti, 2020).

Nesse contexto a ausência de profissionais capazes de auxiliar e esclarecer angústias durante o período gestacional, além de orientar sobre o momento do parto e do puerpério, no qual as mulheres estão sob muita pressão de pessoas que estão 
ao seu redor, e é nesse cenário que o movimento feminista se fez fundamental em 1993, com a inserção da discussão sobre o direito de diálogo sobre o parto, além de ter sido crucial na fundação da Rede pela Humanização do Parto e do Nascimento (Rehuna) (Queiroz et al., 2017).

Nesse sentido Assis (2018) traz que o movimento social feminista negro é fundamental pela luta por direitos e igualdade, no que tange todos os aspectos de vida e principalmente a saúde visto que as mulheres negras são postas à marginalidade e, por esta razão, estão mais vulneráveis a atendimentos sub-humanos (Sena \& Tesser, 2017).

No que diz respeito à atuação profissional, Cobo et al., (2021) deixam evidente que não há preparo na assistência prestada pelos profissionais de saúde, no que diz respeito às questões de gênero, pois desde a formação eles não são instrumentalizados para lidar com problemas de cunho social e, para, além disso, percebe-se a relação dicotômica estabelecida, em que o profissional é detentor do saber (senso comum), e impõe, por uma relação de poder, toda sua "sabedoria" a despeito das vontades e/ou necessidades do seu paciente, que está numa posição de ouvinte (Azeredo \& Schraiber, 2017; Franzoi et al., 2011).

\section{Considerações Finais}

O presente estudo considerou o controle social como um princípio fundamental para a construção de políticas públicas e para a garantia do direito à saúde, dessa forma, o movimento de mulheres se mostrou importante no que se refere a acompanhar e lutar para que tais direitos sejam assegurados.

É importante ressaltar que, para se chegar a uma plena garantia de direitos, há a necessidade de que sejam articulados três pilares: o campo das políticas públicas, responsável por construir os caminhos para a garantia dos direitos, o campo da organização dos serviços, onde os níveis de atenção se farão necessários para a garantia da prática, e o campo das práticas, para que os profissionais reconheçam as suas responsabilidades e as executam de modo a garantir os direitos das mulheres.

A partir da discussão dos temas, identificou-se que os direitos não são plenamente assegurados nos serviços de saúde pois, embora existam políticas que determinam a garantia desses.

Um dos pontos que justificam essa incoerência foi a organização dos serviços, quando estes não existem ou dificultam o acesso do direito, como acontece com os serviços credenciados como garantidores do aborto legal.

Porém, foi identificada como o fator principal a prática cotidiana dos profissionais, na assistência direta às mulheres, que é permeada por preconceitos e julgamentos morais que distanciam o atendimento das práticas éticas e responsáveis, consequentemente não há garantia do direito à saúde das mulheres de modo integral, como é preconizado.

É necessário capacitar os profissionais de saúde exaltando sua responsabilidade na garantia do direito à saúde da população prestando uma assistência ética, para que a partir da compreensão das questões de gênero e suas desigualdades com o objetivo de que as mulheres sejam reconhecidas em sua autonomia e possam participar como protagonistas da sua saúde.

\section{Referências}

Assis, J. F. (2018). Interseccionalidade, racismo institucional e direitos humanos: compreensões à violência obstétrica. Rev. Serv. Soc. Soc., 133, 547-565. $10.1590 / 0101-6628.159$

Azeredo, Y. N., \& Schraiber, L.B. (2017). Violência institucional e humanização em saúde: apontamentos para o debate. Revista ciência e saúde coletiva, 22(9): 3013-22. 10.1590/1413-81232017229.13712017

Bardin. L. (1977). Análise de Conteúdo. Edições 70.

Brasil. (1940). Lei $n^{o}$ 2.848, de 7 de dezembro de 1940. Da Aplicação da Lei Penal. Brasília, Brasil. http://www.planalto.gov.br/ccivil_03/decretolei/Del2848.htm

Brasil. (1990). Lei $n^{\circ} 8.080$, de 19 de setembro de 1990. Dispõe sobre as condições para a promoção, proteção e recuperação da saúde, a organização e o funcionamento dos serviços correspondentes e dá outras providências. Brasília, Brasil. http://www.planalto.gov.br/ccivil_03/leis/18080.htm 
Brasil. (1990). Lei $n^{o} 8.142$, de 28 de dezembro de 1990. Dispõe sobre a participação da comunidade na gestão do Sistema Único de Saúde (SUS) e sobre as transferências intergovernamentais de recursos financeiros na área da saúde e dá outras providências. Brasília, Brasil: Ministério da Saúde. from http://www.planalto.gov.br/ccivil_03/leis/L8142.htm

Brasil. (2004). Política Nacional de Atenção Integral à Saúde da Mulher: Princípios e Diretrizes. Brasília, Brasil: Ministério da Saúde. https://conselho.saude.gov.br/ultimas_noticias/2007/politica_mulher.pdf

Brasil. (2012). Política Nacional de Atenção Básica. Brasília, Brasil: Ministério da Saúde. http://189.28.128.100/dab/docs/publicacoes/geral/pnab.pdf

Brasil. (2015). Ministério da Saúde. Atenção Integral À Saúde de Mulheres Lésbicas e Bissexuais. Brasília, Brasil. https://portalarquivos2.saude.gov.br/images/pdf/2015/agosto/31/livreto-atencao-a-saude-de-mulheres-lesbicas-versao-web.pdf

Brasil. (2016). Protocolos da Atenção Básica: Saúde das Mulheres. Brasília, Brasil: Ministério da Saúde. https://bvsms.saude.gov.br/bvs/publica coes/prot ocolos_a tencao_basica_saude_mulheres.pdf

Busin, V. M. (2013). Direitos Humanos para Ativistas por Direitos Sexuais e Reprodutivos. Brasília, Brasil: Secretaria de políticas para Mulheres. http:// www.bib liotecadigital.abong.org.br/bitstream/handle/11465/307/CDD-BR_direitos_humanos_ativistas_direitos_sexuais.pdf?sequence=1

Cardoso, B. B., Vieira, F. M. S. B., \& Saraceni, V. (2020). Aborto no Brasil: o que dizem os dados? Cad. Saúde Pública, 36(sup 1):e00188718. $10.1590 / 01002-311 X 00188718$

Cobo, B., Cruz, C., \& Dick, P. C. (2021). Desigualdade de gênero e raciais no acesso e uso dos serviços de atenção primária à saúde no Brasil. Ciência \& Saúde Coletiva, 26(9): 4021-4032. 10.1590/1413-81232021269.05732021

Costa, A. M. (2009). Participação Social na Conquista das Políticas de Saúde para Mulheres no Brasil. Ciência e Saúde Coletiva, 14(4), 1073-1083. $10.1590 /$ S1413-81232009000400014

Diniz, D., Dias, V. C., Mastrella, M. \& Madeiro, A. P. (2014). A verdade do estupro nos serviços de aborto legal no Brasil. Rev. Bioét, 22(2), 291-8. $10.1590 / 1983-80422014222010$

Diniz, D., Corrêa, M., Squinca, F. \& Braga, K. S. (2009). Aborto: 20 anos de pesquisas no Brasil. Cad. Saúde Pública, 25(4), 939-942. 10.1590/S0102311 X2009000400025

Bourguignon, A. M., \& Grisotti, M. (2020). A humanização do parto e nascimento no Brasil nas trajetórias de suas pesquisadoras. Hist. Cienc. SaúdeManguinhos, 27(2), 485-502. 10.1590/S0104-59702020000200010

Franzoi, N. M., Fonseca, R. M. G. S. \& Guedes, R. N. (2011). Violência de gênero: concepções de profissionais das equipes de saúde da família. Revista Latino Americana Enfermagem. 19(3), 9 telas. 10.1590/S0104-11692011000300019

Santos, J. S., Silva, R. N. \& Ferreira, M. A. (2019). Saúde da população LGBTI+ na atenção primária à saúde e a inserção da enfermagem. Esc. Anna Nery. 23(4), e20190162. 10.1590/2177-9465-EAN-2019-0162

Paradis, C. (2014). A luta política feminista para despatriarcalizar o Estado e construir as bases para a igualdade. In: Moreno, R. (0rg) Feminismo, economia e política. Debates para a construção da igualdade e autonomia das mulheres. São Paulo: SOF Sempreviva Organização Feminista. http://www.sof.org.br/wpcontent/uploads/2015/08/Economia-e-poli\%CC\%81tica-web.pdf

Madeiro, A. P., \& Diniz, D. (2016). Serviços de aborto legal no Brasil - um estudo nacional. Ciência \& Saúde Coletiva, 21(2): 63-572. 10.1590/141381232015212.10352015

Medeiros, J. M. M. (2021). Desafios à política de saúde brasileira: impactos no direito ao aborto legal. Rev. Katálysis, 24(2): 280-290. 10.1590/19820259.2021.e75661

Queiroz, T. C., et al. (2017). Violência Obstétrica e suas perspectivas na relação de gênero. Rev. Científica Fagoc Saúde. http://revista.fagoc.br/index.php/saude/article/view/194

Saffioti, H. I. B. (2011). Gênero, patriarcado, violência. São Paulo/SP: Editora Fundação Perseu Abramo. https://edisciplinas.usp.br/pl uginfile.php/17 41437/ mod_resource/content/1/Gênero, Patriarcado, Violência \%28livro completo\%29.pdf

Saffioti, H. I. B. (1997). Violência de género: o lugar da práxis na construção da subjetividade. Revista Lutas Sociais: 59-79. http://www4.pucsp.br/neils/downloads/v2_artigo_saffioti.pdf

Santos, A. E. A., Leão, F.M., Araújo, L. S., \& Ferreira, L.T.H. (2010). Sexual e Reprodutiva: direitos e desafios em um mundo multicultural. In: Liberdade identitária como Direito Fundamental. http://www.sinus.org.br/2011/press/downloads/oms.pdf

Santos BS. (1987). O Estado, a Sociedade e as Políticas Sociais. Revista Críticas de Ciências Sociais; 1987. 23: 13-73. http://www.boaventu radesousasa ntos.pt/media/pdfs/Estado_Sociedade_Politicas_Sociais_RCCS23.PDF

Sena, L. M., \& Tesser, C. D. (2017). Violência Obstétrica no Brasil e o Ciberativismo de mulheres mães: relato de duas experiências. Rev. Interface comunicação, saúde, educação, 21(60):209-20. http://www.scielo.br/pdf/icse/v21n60/1807-5762-icse-1807-576220150896.pdf

Silva, S. G. (2013). La conferencia de El Cairo y la afirmación de los derechos sexuales y reprocuctivos, como base para la salud sexual y reproductiva. Rev Peru Med Exp Salud Publica, 30(3): 455-60. http://www.scielo.org.pe/pdf/rins/v30n3/a14v30n3.pdf

Souto, K., \& Moreira, M. R. (2021). Política Nacional de Atenção integral à Saúde da Mulher: protagonismo do movimento de mulheres. Saúde e Debate, 45(130): 832-846. 10.1590/0103-1104202113020 
Research, Society and Development, v. 10, n. 14, e137101421826, 2021

(CC BY 4.0) | ISSN 2525-3409 | DOI: http://dx.doi.org/10.33448/rsd-v10i14.21826

Talib, R. A., \& Citeli, M. T. (2005). Serviços de aborto legal em hospitais públicos brasileiros, (1989-2004) Dossiê. Católicas pelo Direito de Decidir. http://catolicas.org.br/wp-content/uploads/2005/01/Caderno-DossieAbortoLegal.pdf

Tesser, C. D., Knobel, R.., Andrezzo, H. F. A., \& Diniz, C. S. G. (2015). Violência obstétrica e prevenção quaternária: o que é e o que fazer. Rev Bras Med Fam Comunidade, 10(35):1-12. 10.5712/rbmfc10(35)1013

Weidle, W. G., Medeiros, C. R. G., Grave, M. T. Q., \& Bosco, S. M. D. (2014). Escolha da via de parto pela mulher: autonomia ou indução? Cad. Saúde Coletiva, 22(1): 46-53. 10.1590/1414-462X201400010008

Tavares, V. M. C., Neto, L. M. A, Pereira, E. S, Taveira, M. G. M. M;. \& Cavalcante, J. K. (2020). Roda de conversa: atenção integral à saúde das mulheres e questões de gênero. Brazilian Journal of Development, 6 (8), 61501- 61510. 10.34117/bjdv6n8-537 